\title{
Los matrimonios mixtos en España ¿espacios de construcción intercultural?
}

\author{
MARIA CARMEN ALBERT GUARDIOLA \\ IUDESP. UNIVERSIDAD DE ALICANTE \\ ERIKA MASANET RIPOLL \\ IUDESP. UNIVERSIDAD DE ALICANTE
}

\section{Resumen}

El objetivo del artículo es plantear el contexto teórico de las características que envuelven el fenómeno de las familias multiculturales, es decir, aquellas familias en las que el origen étnico o la primera nacionalidad de uno de los miembros de la pareja no es la española (también llamadas parejas o matrimonios mixtos). Se trata de analizar las consecuencias que se derivan de la diversidad cultural, más allá de la cuantificación del hecho migratorio y presentar la metodología del estudio financiado por el Ministerio de Educación y Ciencia.

Palabras clave: matrimonio mixto, familia multicultural, identidad, socialización

\section{Abstract}

The aim of this paper is to present the theoretical context of multicultural families, that is, families in which ethnicity or first nationality of one of the partners is not the Spanish (also called couples or mixed marriages). The text analyses the consequences of cultural diversity, beyond of migration quantification and present the methodology of the study funded by the Ministry of Education and Science.

Keywords: Mixed Marriage, multicultural family, identity, socialization. 


\section{Introducción}

La inversión en el panorama migratorio español se produce en la década de los ochenta, sobre todo a raíz de la adhesión a la Comunidad Económica Europea (CEE) en 1986. En este contexto, durante los noventa se asiste a una importante afluencia de inmigrantes procedentes de un abanico geográfico, cultural y étnico cada vez más amplio que conduce hacia una creciente diversificación nacional y étnica de los flujos migratorios establecidos en España.

Las cifras dan cuenta del cambio social de gran magnitud que se ha producido en la sociedad española en poco tiempo. Así lo evidencia el hecho de que la población extranjera empadronada haya crecido desde 1996 hasta el 2007 en casi 4 millones de personas (INE, 2007).

El estado español se consolida a finales de los noventa como país de inmigración y pasa a convertirse en un espacio multicultural y pluriétnico, con las implicaciones de diversa índole (económicas, sociales, políticas y culturales) que esto conlleva. En este sentido, uno de los efectos del incremento de la población extranjera en el estados español es a su vez, el aumento en el número de los matrimonios o de la parejas mixtas y la consecuente formación de familias multiculturales o transnacionales (esta última denominación es usada por Rodríguez, 2004a y 2004b). Este fenómeno muestra "como cambian los modos de construcción de la familia en la emigración y cuáles son las formas que asume la interacción y el encuentro con el otro" (Tognetti Bordogna, 1996: 9). Se trata de una realidad heterogénea, analizada mucho más en referencia a los aspectos de la problemática y del conflicto que en aquellos que tratan la creatividad y la innovación (Varro, 1995).

En la literatura europea como en la norteamericana sobre migraciones y relaciones interétnicas, se utiliza el término "matrimonio mixto" como equivalente de "bi-nacional" (Rodríguez, 2004a: 79). Asimismo, es habitual en la literatura anglosajona el empleo del término "matrimonios interraciales" para hacer alusión a diferencias fenotípicas, y el de "matrimonios interétnicos" para referirse al matrimonio entre personas de culturas diferentes, aunque estos dos espacios suelen mezclarse. En los Estados Unidos y el Reino Unido los matrimonios interétnicos también se suelen denominar matrimonios mixtos ("mixed marriages") (Rodríguez, 2004b: 51). 
Las variables o criterios de clasificación utilizados para definir un matrimonio mixto pueden ser diversos: país de origen, país de nacimiento, nacionalidad, grupo étnico, religión, rasgos fenotípicos, idioma, etc. (Crester 1990: 229). De ahí la necesidad de especificar la variable a la que nos referimos cuando hablamos de patrones matrimoniales o uniones mixtas. En nuestro caso, los matrimonios mixtos objeto de nuestro estudio son aquellos en los que la primera nacionalidad de uno de los cónyuges es diferente a la española, lo que implica, en general, un origen cultural distinto. A este respecto, Rodríguez (2004b: 51) sostiene que si nos referimos a matrimonios entre personas de distinto país, sería mejor hablar de "matrimonios mixtos según el país", "bi-nacionales" o "inter-nacionales". De hecho, en la literatura inglesa y americana se usa cada vez más el término "intercountry marriage" o "bi-national marriage" para hablar de uniones mixtas.

Según datos del Anuario Estadístico de Extranjería 2006, en el 2005 se registraron en España un total de 29.925 matrimonios con al menos uno de los cónyuges de origen extranjero, lo que representa el $14,3 \%$ del total de los matrimonios (209.415). En relación con los datos del 2004 no existen apenas variaciones significativas, sin embargo se ha producido un incremento desde el 2002 en el número de matrimonios en los que al menos uno de los contrayentes era extranjero. Cabe señalar que un $24 \%$ del total de estos matrimonios celebrados en el 2005 corresponden a enlaces entre mujer y varón extranjero. En definitiva podemos decir que los matrimonios mixtos, aquellos en los cuales un cónyuge era extranjero y otro español es de: $22.402(76 \%)$. Asimismo, el porcentaje de hombres casados con extranjeras es mayor que el de mujeres.

Respecto a la Comunidad Autónoma de residencia del matrimonio según los datos del Anuario 2006, el 21\% de los que se celebraron con al menos un cónyuge extranjero residía en Cataluña, el $17 \%$ en la Comunidad de Madrid, el 13\% en la Comunidad Valenciana y el $12 \%$ en Andalucía. De este modo, la distribución de los matrimonios mixtos en el Estado español se corresponde con la concentración geográfica de la población extranjera en determinadas comunidades autónomas. Lógicamente, las zonas con mayor proporción de población extranjera permiten más oportunidades para 
la interacción social de diferentes grupos en diversos ámbitos (trabajo, estudios, etc.).

En cuanto a la forma de celebración del matrimonio, una gran mayoría de los matrimonios mixtos fueron exclusivamente civiles, el $81 \%$, el $18 \%$ se celebraron según el rito católico y menos del 1\% según otra religión.

Según el continente de origen de los contrayentes en los matrimonios mixtos, las mujeres españolas contrajeron matrimonio en el 2005 mayoritariamente con ciudadanos de países de América Latina $(36 \%)$, seguido muy de cerca de ciudadanos de países europeos, principalmente de la Europa comunitaria y africanos (22\%). Respecto a la nacionalidad de los extranjeros que se casaron con mujeres españolas, el colectivo más numeroso fue el de varones argentinos, colombianos y ecuatorianos, y el de rumanos e italianos en lo que concierne a países europeos, finalmente en el caso de países del continente africano destacan marroquíes y nigerianos.

El objetivo de este artículo es plantear el contexto teórico de las características que envuelven el fenómeno de las familias multiculturales, familias formadas por parejas o matrimonios mixtos: aquellas parejas en las cuales el origen étnico o la primera nacionalidad de uno de sus miembros no es española ${ }^{1}$. Se trata de analizar en profundidad las consecuencias que se derivan de la diversidad cultural, más allá de la cuantificación del hecho migratorio y presentar la metodología del estudio financiado por el Ministerio de Educación y Ciencia (SEJ2005-05034)

\section{Interacciones culturales: Multiculturalismo interculturalidad en las parejas mixtas}

La diversificación y la producción investigadora sobre migraciones en nuestro país es abundante. Los análisis de este fenómeno proceden desde las más variadas disciplinas y enfoques. Sin embargo, los estudios realizados en nuestro país raras veces han

\footnotetext{
1 Aunque como se podrá comprobar en los siguientes apartados, la definición de parejas mixtas viene definida en mayor proporción a través de la condición de matrimonio, en este trabajo se ha pretendido dimensionarlo como familia multicultural, independientemente del estado civil de los miembros de la unidad familiar, el único requisito es la convivencia.
} 
abordado los contextos multiculturales y las dinámicas de las relaciones interculturales en el ámbito específico de la formación de la pareja y los matrimonios mixtos, cuando este fenómeno, tal y como señala Dan Rodríguez, constituye uno de los ámbitos más fundamentales en los procesos de interculturalidad (2004a: 79). Es más, la unidad familiar se configura como un motor de cambio social en contextos multiculturales, de ahí la importancia de este nuevo fenómeno.

Así, los estudios sobre la formación de matrimonios mixtos y de identidades multiculturales de los inmigrantes e hijos de inmigrantes -segundas generaciones- han crecido notablemente en el Reino Unido, Francia, Estados Unidos o Canadá, mientras que en los países del sur de Europa (Italia y España), este fenómeno ha empezado a ser estudiado recientemente (Tognetti-Bordogna, 1996; Rodríguez, 2004a; Bermúdez, 2007).

En los Estados Unidos el incremento de los matrimonios mixtos constituye una de las tendencias demográficas más importantes en el país. Dicho aumento de los matrimonios mixtos es uno de los factores clave que ha contribuido al incremento de la diversidad racial y étnica de la población de los EEUU en décadas recientes (Lee y Edmonston, 2005: 11). En efecto, el aumento de matrimonios mixtos y consecuentemente de familias multiculturales ha dado lugar al crecimiento de grupos de población con múltiples orígenes étnicos (Sandefur, 1986; Perlmann, 1997; Golstein, 1999).

Una de las consecuencias sociales de los matrimonios mixtos, según Lee y Edmonston (2005), es la reducción de la distancia social entre los grupos raciales, así como el cambio del significado del concepto de 'raza'.

Dos son los ámbitos y contextos a partir de los cuales tienen lugar los procesos que se quieren analizar en nuestro estudio: la interculturalidad y multiculturalismo. Ambos términos, aunque están estrechamente relacionados entre sí, presentan realidades distintas como bien señalan algunos autores (Perotti, 1989; Kymlicka, 1996; Rodrigo, 1997; Walsh, 2001).

2 El termino 'raza' es la terminología utilizada por los investigadores estadounidenses, mientras que en el contexto europeo se hace referencia a 'étnico' por las connotaciones negativas que ofrece la primer caso. 
Para muchos teóricos actuales, el multiculturalismo es una propuesta sociopolítica que respeta las diversidades culturales existentes en su seno y el derecho a la diferencia, desde la tradicion liberal (Taylor, 1993) ${ }^{3}$. Recientemente, estos principios básicos como tales han sido objeto de críticas, ya que se hace referencia a la idea de la suma de culturas y de coexistencia (Kymlicka, 1996; Rodrigo, 1997). En otras palabras, la multiculturalidad se refiere a "la multiplicidad de culturas que existen dentro de un determinado espacio, sea local, regional, nacional o internacional" (Walsh, 2001: 5). Por el contrario, la interculturalidad -concepto relativamente nuevo- se refiere a las "complejas relaciones, negociaciones e intercambios culturales, y busca desarrollar una interacción entre personas, conocimientos y prácticas culturales diferentes; una interacción que reconoce y que parte de las asimetrías sociales, económicas, políticas y de poder y de las condiciones institucionales que limitan que el "otro" pueda ser considerado como sujeto con identidad, diferencia y agencia la capacidad de actuar" (Walsh, 2001: 6).

En resumen, mientras que la multiculturalidad consistiría en la mera coexistencia dentro de una misma sociedad de diversas culturas, la interculturalidad iría más allá e implicaría la interacción e interrelación entre grupos y minorías étnicamente diferenciadas buscando la participación y los elementos positivos de una y otra.

Desde este punto de vista, en el caso de las parejas mixtas como bien señala Dan Rodríguez "el contexto de formación y dinámica de las uniones mixtas constituye un espacio de hibridez sociocultural especialmente activo y complejo; y que estas dinámicas implican procesos de negociación y acomodación de bagajes socioculturales diversos" (2004a: 114). Es aquí donde entran en consideración aspectos tan importantes como la socialización y la conformación de identidades culturales múltiples o hyphenadas (Therborn, 1995).

3 Para un desarrollo teorico sobre el multiculturalismo como modelo socio-politico y un analisis de los diversos modelos, veáse Delanty (2003). 


\section{Consideraciones teóricas sobre las uniones mixtas}

\section{Los patrones en la elección del cónyuge}

La literatura sobre los matrimonios mixtos se articula principalmente en torno a dos temas: los patrones y/o los factores en la elección del cónyuge y las consecuencias de los matrimonios mixtos (Kalmijn, 1998: 396).

Tanto los estudios teóricos como los empíricos han desarrollado hipótesis sobre las razones por las que la gente se casa dentro de su grupo, mientras que otros lo hacen fuera de su propio grupo. En este sentido, las teorías sobre la elección de la pareja han proporcionado importantes claves sobre las causas del matrimonio mixto y la homogamia (Kalmijn, 1998). Tal y como sostiene este último autor (Kalmijn, 1991 y 1998), en general, los patrones o modelos matrimoniales surgen de la interacción entre tres fuerzas sociales: las preferencias individuales para ciertas características en el cónyuge, la influencia del grupo social del que son miembros y las limitaciones del mercado matrimonial en que ellos buscan un cónyuge.

Para Dan Rodríguez (2004b: 27), el proceso de elección del cónyuge y la formación del matrimonio y la familia es un fenómeno multidimensional que viene determinado por diferentes factores, todos ellos interrelacionados. El autor agrupa los distintos factores de influencia en la elección de la pareja en cuatro tipos de factores determinantes, cada uno de ellos influenciados por la libre elección.

En cuanto al grupo de los factores demográficos, la elección de la pareja o cónyuge y los procesos de endogamia/ exogamia se producen dentro de la estructura demográfica de población y de los 'mercados matrimoniales' (Rodríguez, 2004b: 31-32). A pesar de enfatizar la importancia de los determinantes puramente demográficos (estado civil y edad principalmente), cabe considerar también el factor de la distancia territorial, así como factores socioculturales e individuales en estos procesos: el estatus socioprofesional, la nacionalidad, el nivel de estudios, la religión, etc. (Girard, 1981; Musham, 1974).

Respecto a los factores psicosociales, las percepciones, actitudes y comportamientos individuales en torno a la elección de la pareja están en gran medida influidos y vinculados al concepto de 
"endo-grupo" y exo-grupo", es decir, "a la imagen que un individuo tiene de sí mismo en tanto que miembro de ese grupo/s, y de la de los demás, en tanto que miembros del mismo/s o diferente/s grupo/s" (Rodríguez, 2004b: 37).

Figura 1. Factores de influencia en la elección cle la pareja

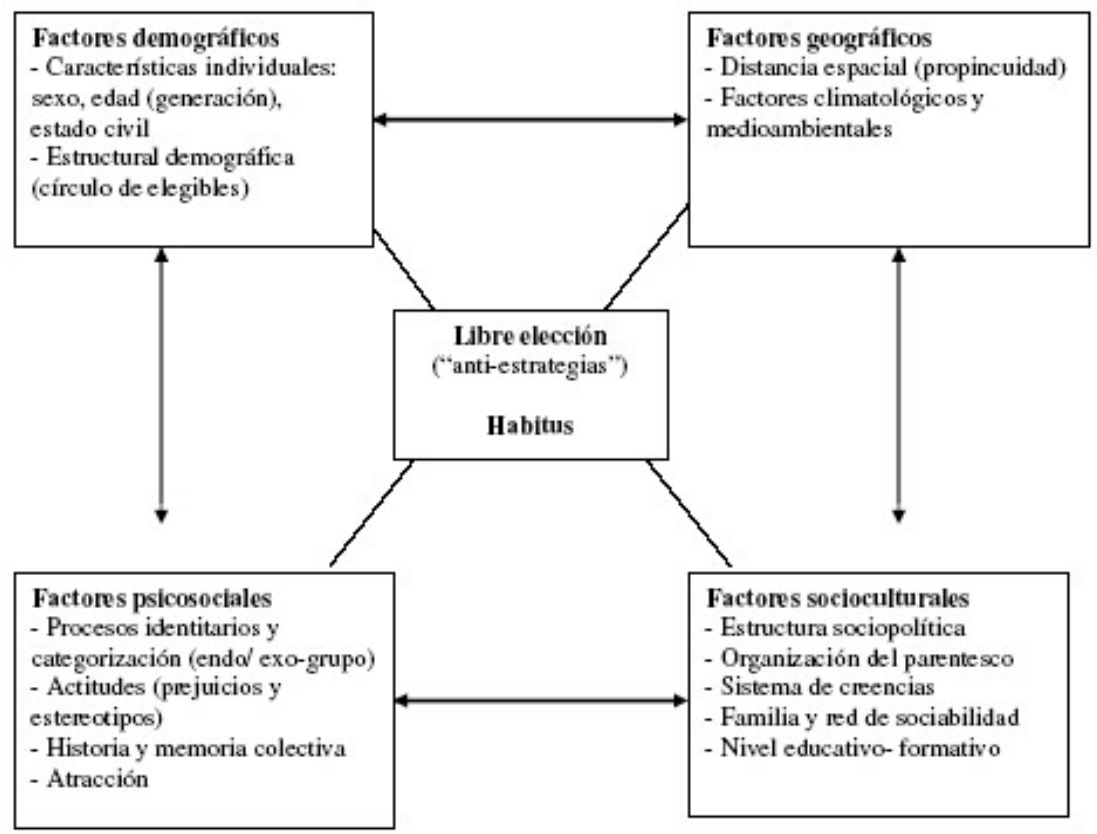

Nota: las flechas más gruesas indican mayor imbricación entre los factores; y el hecho de que la "libre elección" ocupe el centro del diagrama no significa que se le conceda más importancia, sino su existencia en conexión con todos los factores.

Fuente: Rodrignez (2004b: 28).

Pasamos a centrarnos en los factores sociológicos o el marco social de la elección de la pareja. Desde la sociología, las teorías de la elección matrimonial en las sociedades urbanas, industriales o postindustriales han sido ampliamente desarrolladas. Los "matrimonios de amor", en general más exógamos, son más frecuentes cuanto más urbana sea la sociedad. Es decir, el proceso de elección de la pareja o cónyuge es más abierto en las sociedades urbanas modernas. Sin embargo, no existe la pretendida libertad total en la elección de la 
pareja en las sociedades complejas, puesto que como afirman Zonabend (1981) y Carabaña (1983) ${ }^{4}$, "nos movemos en redes de relación determinadas y nos casamos con personas no demasiado próximas, pero tampoco demasiado lejanas". La proximidad en las sociedades urbanas modernas estaría conformada por la ocupación, el estatus u otra variable que implique afiliación o ámbito de relación. De este modo, en la elección de la pareja se siguen "estrategias individuales, inducidas por consideraciones sociales y económicas" (Rodríguez, 2004b: 33-34).

La relevancia del factor social en las uniones o matrimonios y, por tanto, el papel decisivo del estatus económico y socioprofesional en los procesos de endogamia/ exogamia se pone de manifiesto en los estudios llevados a cabo para tal fin. Incluso en las uniones interétnicas, es un hecho el predominio de la homogamia (Kalmijn, 1998; Fu, 2001; Rodríguez, 2004a y 2004b; Lee y Edmonston, 2005).

El conjunto de teorías a nivel microestructural sobre la selección matrimonial muestran al matrimonio como una entidad o espacio que produce comodidades como los hijos, el estatus, la seguridad, el soporte económico y el soporte social (Becker, 1991: 24; Kalmijn, 1998; Oppenheimer, 1997). En este contexto, para comprender los modelos de la selección matrimonial, los investigadores suelen usar el concepto de "mercado matrimonial" (Kalmijn, 1998: 398).

Este paradigma del "mercado matrimonial" se basa en la metáfora del mercado para explicar el proceso de selección matrimonial: los hombres y mujeres solteros actúan dentro de un mercado matrimonial donde cada individuo considera un conjunto de cónyuges potenciales. En este mercado matrimonial la gente "compra" el mejor cónyuge que pueden atraer con los recursos que ellos pueden ofrecer. Cuando se casan, los cónyuges juntan estos recursos para producir bienes o productos familiares como el bienestar económico, el estatus, la confirmación social y el afecto. Existen distintos tipos de recursos que intervienen en la elección del cónyuge, pero los sociólogos se centran sobre todo en los recursos socioeconómicos y culturales (Kalmijn, 1998: 398).

\footnotetext{
${ }^{4}$ Citado en Rodríguez, 2004b: 33.
} 
Los recursos socioeconómicos se definen como aquellos recursos que producen bienestar económico y status. La gente maximiza su renta y estatus buscando un cónyuge con unos recursos socioeconómicos atractivos. La competencia por los recursos socioeconómicos en el mercado matrimonial conduce a unos patrones agregados de homogamia. Por otro lado, los recursos culturales se basan en las preferencias por la similaridad cultural que se puede producir en tres tipos de niveles: a) la similaridad de los valores y opiniones, que conlleva a una confirmación mutua del comportamiento y la cosmovisión de cada uno; b) la similaridad de gustos, que se torna atractiva porque incrementa las oportunidades de participar en actividades conjuntas; y, c) la similaridad del conocimiento, que crea una base común para la conversación, mejorando el entendimiento mutuo. De este modo, la similaridad cultural conduce a la atracción personal y también anima a la gente a establecer relaciones a largo plazo (Kalmijn, 1998: 398-399).

Según Murstein (1976), la selección del cónyuge se trataría de un proceso filtrado. En un primer paso, la gente desarrolla una red de amigos, conocidos y posibles candidatos al matrimonio con quienes comparte algunas características sociales objetivas. En la segunda etapa, la gente encuentra su cónyuge interactuando dentro de esa red homogénea. En esta segunda fase las características psicológicas juegan un papel importante, pero al mismo tiempo la homogamia con respecto a las características sociales objetivas ya están aseguradas.

La teoría del mercado matrimonial nos ayuda a entender por qué las parejas a menudo comparten una variedad de características. Dicha combinación de características puede ser el patrón dominante, pero los recursos pueden ser intercambiados cuando la combinación no es perfecta. Asimismo, el enfoque del mercado matrimonial se fundamenta en dos premisas básicas sobre los matrimonios mixtos. La primera consiste en que los individuos con altos niveles de recursos en general pueden atraer cónyuges con similares niveles de recursos. La segunda idea se basa en que las parejas no combinan en todos los recursos (Fu, 2001: 148). Aplicando estos dos principios para los matrimonios mixtos, $\mathrm{Fu}$ (2001: 148-149) establece tres tipos de escenarios que describen cómo diferentes situaciones de partida pueden afectar a las características de los matrimonios mixtos: 
1. El primer escenario es el de la perspectiva de los matrimonios mixtos endogámicos, el cual sostiene que los matrimonios mixtos siguen los mismos patrones que los matrimonios endogámicos. Según esta perspectiva, los matrimonios mixtos entre miembros de diferentes grupos se producen entre personas con similar nivel educativo.

2. El segundo escenario es el de la hipótesis del intercambio del estatus originada por Davis (1941) y Merton (1941). Según estos autores, cuando las personas pertenecientes a minorías forman matrimonios mixtos compensan a sus cónyuges con otros recursos como pueden ser los estudios. Por ejemplo, los matrimonios mixtos entre hombres negros con altos niveles educativos y mujeres blancas con menores niveles educativos podrían ser un patrón común. En estos casos, el alto nivel educativo de los hombres negros compensaría su bajo status racial o étnico. El modelo de Davis y Merton sugiere que los miembros de grupos étnicos cuyo prestigio en la sociedad es menor, tienen más oportunidades de exogamia si ofrecen un alto estatus socioeconómico a cambio. La hipótesis del intercambio, según Kalmijn (1998: 416), también tiene su aplicación para otras dimensiones de la elección de la pareja, como el atractivo físico y la participación cultural. Según este enfoque, los patrones de los matrimonios mixtos reflejan la jerarquía racial generalizada.

3. El tercer escenario sugiere que los patrones de los matrimonios mixtos reflejan una preferencia por los cónyuges pertenecientes al propio grupo. Bajo esta perspectiva in-group preference, la gente se casa fuera de su propio grupo solamente cuando no pueden encontrar un cónyuge deseable que pertenezca a su propio grupo. Desde esta perspectiva, la similitud es atractiva en la interacción social y a su vez, la similitud cultural permite a la pareja forjar una vida en común y realza la ayuda mutua. Siguiendo este enfoque, los individuos con más estudios tendrán más probabilidades de casarse dentro de su propio grupo.

Las personas solteras no sólo viven y actúan en una determinada región buscando cónyuge, sino que en el mercado matrimonial hay una serie de escenarios funcionales donde las personas solteras pasan la mayor parte de sus vidas como el vecindario, la escuela, el lugar de trabajo, los bares, los clubs, etc... En la literatura 
sociológica los tres lugares del mercado matrimonial que han sido más frecuentemente estudiados han sido la escuela, el vecindario y el lugar de trabajo. Estos pequeños y funcionales locales del mercado matrimonial influyen en la homogamia (Kalmijn, 1998: 403). Así, la función de las instituciones educativas como mercado matrimonial favorece la homogamia educacional. Mientras que en los escenarios en que buscan una pareja en una etapa posterior, como el trabajo o los lugares públicos, tienden a ser menos homogéneos (Kalmijn, 1998: 414).

Tradicionalmente se ha considerado la endogamia como un comportamiento que limita la integración pluriétnica y mantiene las diferencias, en contraposición con la exogamia que tiende a favorecer la integración y que presupone la ausencia de prejuicios étnico-raciales (Coleman, 1994). Esta afirmación también es secundada hoy en día por algunos estudiosos norteamericanos como Lee and Edmonston (2005: 4), según los cuales los matrimonios "interraciales" son un indicador particularmente significativo de la asimilación o integración de las minorías étnicas.

Sin embargo, estudios anteriores como el estudio clásico de Bastide (1961) y otros más recientes como el de Lievens (1999) y Fu (2001) señalan que, aunque algunos matrimonios mixtos pueden facilitar la integración social del grupo, éstos no indican una aceptación completa entre miembros de los dos grupos y que por tanto, el mestizaje no entraña ausencia de prejuicios.

Desde este punto de vista, el matrimonio exógamo no significa necesariamente un comportamiento 'moderno' o la panacea de la integración, ni el matrimonio endógamo una opción negativa o un signo de comportamiento 'tradicional'. Estas consideraciones acerca de la exogamia y endogamia, por tanto, deben ser cuestionadas (Rodríguez, 2004). Tal y como señala este último autor (2004: 84), el matrimonio mixto no es signo de integración si, por ejemplo, un inmigrante se casa con un individuo nativo porque el Gobierno dificulta la reunificación con su esposa/o. En este sentido, un incremento en el número de matrimonios mixtos puede deberse en parte a un marco legislativo inmigratorio restrictivo.

Como ya mencionamos anteriormente, los estudios sobre la conformación de los matrimonios mixtos y los procesos de 
endogamia/ exogamia en el caso español son más bien escasos. La única investigación encontrada es la tesis doctoral de Dan Rodríguez (2004), cuyo tema central es la formación de la pareja y la familia y los procesos de endogamia/ exogamia de la población africana (particularmente de Senegal y Gambia) residente en Cataluña.

Los resultados de la investigación de Rodríguez con inmigrantes africanos muestran el predominio de la endogamia (matrimonio dentro del propio grupo/ categoría o misma nacionalidad) y la homogamia (matrimonio entre individuos de similar estatus social), independientemente del país y región de nacimiento-socialización, religión, grupo étnico-lingüístico, familia (vínculo parentesco) y estatus social (2004a: 91). Estos datos confirman las conclusiones a las que llegan múltiples estudios realizados desde una variedad de disciplinas, acerca de la preeminencia de las tendencias de la endogamia y la homogamia en los contextos de migración (Merton, 1941; Winch, 1958; Musham, 1974; Murstein, 1986; Gray, 1987; Coleman, 1994; Varro, 1995; Kalmijn, 1998; Fu, 2001) ${ }^{5}$.

En relación con la homogamia, una de las mayores aportaciones del estudio de Rodríguez (2004) es su tesis sobre la importancia del factor social en los procesos de endogamia /exogamia en detrimento del factor cultural, de tal modo que la homogamia socioeconómica es la que predomina tanto en las uniones endógamas como en las exógamas. En palabras del autor, "la clase social parece ser un factor más explicativo que el origen geográfico-cultural para explicar los patrones de endogamia y exogamia, así como las dinámicas de convivencia y crianza de los hijos en las uniones mixtas" (Rodríguez, 2004: 114).

Los investigadores han examinado una serie de variables que influyen en la probabilidad de los matrimonios mixtos (Kalmijn, 1998; Rodríguez, 2004; Lee y Edmonston, 2005, etc.). Las variables básicas analizadas han sido la educación, el sexo y la región, observando diferencias en cada una de ellas (Kalmijn, 1998: 412). En cuanto a los efectos de la variable educación, muchos estudios llegan a la conclusión de que los miembros de los grupos minoritarios étnicos con alta educación se casan más exogámicamente que los que tienen menos

\footnotetext{
${ }^{5}$ Citado en Rodríguez (2004: 91).
} 
estudios. Las causas se encuentran tanto en las oportunidades como en las preferencias (Kalmijn, 1998: 413). Respecto a la variable sexo, las diferencias entre hombres y mujeres en cuanto a la probabilidad del matrimonio mixto varía según el grupo al que pertenezcan. A este respecto influyen los roles del hombre y la mujer dentro del grupo étnico y las relaciones sociales que se establecen dentro del grupo. En tercer lugar, la composición étnica de la población de un área puede influenciar en los niveles de los matrimonios mixtos de la región. Así, los matrimonios mixtos tienen más probabilidad de residir en áreas más populosas y en donde la población es étnica y culturalmente diversa (Lee y Edmonston, 2005).

Asimismo, hay otra serie de factores que contribuyen al matrimonio mixto. La variable edad sería una de ellas. Así, las generaciones jóvenes (los inmigrantes más jóvenes y los descendientes de inmigrantes) tienden a ser más exógamas que las generaciones viejas, debido a la desvinculación con el país de origen propio o de los padres y a la consecuente diversificación de las redes sociales (Rodríguez, 2004). El estudio de Lee y Edmonston (2005) además de analizar la influencia del nivel educativo, el sexo y el lugar de residencia, también muestra la importancia de otras variables que pueden afectar a la formación de los matrimonios mixtos como es el tamaño del grupo y el lugar de nacimiento (nacimiento o no en el país de acogida). El tamaño del grupo tiene un importante efecto sobre la probabilidad de que sus miembros se casen fuera del grupo, siendo más probable el matrimonio mixto entre las poblaciones pequeñas (Lee y Edmonston, 2005: 12). De este modo, en general, la gente joven, con altos estudios y nacidos en EEUU están asociados a una mayor probabilidad de matrimonios mixtos, pero esto puede variar según el grupo étnico de pertenencia del hombre y de la mujer (Lee y Edmonston, 2005: 15).

\section{Los efectos de los matrimonios mixtos}

Dos son los efectos de los matrimonios mixtos. En primer lugar, los matrimonios mixtos disminuyen las distinciones culturales en las futuras generaciones porque los hijos de los matrimonios mixtos tienen menos probabilidad de identificarse con un solo grupo. Y, en segundo lugar, la relación íntima que se establece en el matrimonio o pareja puede debilitar las actitudes negativas, prejuicios y estereotipos hacia otros grupos (Kalmijn, 1998: 396). 
La unión o matrimonio mixto se configura como un espacio complejo en que tienen lugar procesos de negociación y de adaptación. Frente a las alternativas costumbres, roles, normas e ideologías ofrecidas en el matrimonio mixto, se puede llevar a cabo un proceso de elección y de incorporación creativa. Se trata de un proceso que Breger y Hill (1998) han denominado como "bricolaje cultural". Según estas autoras, este proceso permite a la familia escoger aquellas partes de cada tradición cultural que ellos quieren mantener, construyendo así una micro-identidad a través de la negociación de las costumbres, prácticas, rituales e identidades de ambas culturas que deseen incluir en la nueva familia (Breger and Hill, 1998: 19-20).

Tal y como señala Dan Rodríguez (2004b: 162), "el contexto por excelencia en el que tienen lugar todas estas dinámicas de adaptación y negociación, de hibridez cultural, es en la educación de los hijos". Del mismo modo y como apuntan Santelli y Collet (1998: 67), los hijos son el corazón de las dinámicas familiares y revelan las decisiones relativas a la vida en pareja. Asimismo, la llegada de los hijos y las prácticas que resultan del espacio familiar constituyen un buen análisis de los vínculos entre el espacio privado, íntimo y el espacio público, exterior (Santelli y Collet, 1998: 74).

Un tema central en el estudio de las dinámicas conyugales en relación con los hijos de los matrimonios mixtos, gira en torno a la cuestión de la identidad. Al igual que sucede con los padres, los hijos de los matrimonios mixtos también deben escoger la incorporación de ciertas características provenientes de las tradiciones culturales de las que son herederos, lo que conlleva a la existencia de una pluralidad de identidades o multi-etnicidades (Breger and Hill, 1998: 23). En este sentido, Rodríguez (2004a: 116; 2004b: 185) resalta la importancia de que las segundas generaciones mantengan 'competencias biculturales' para el aprovechamiento de los recursos y oportunidades disponibles con el fin de conseguir una adaptación exitosa. Sin embargo, la elección de la identidad de los hijos mixtos raramente es para siempre, sino que puede cambiar con los ritmos y las progresiones del ciclo de vida y del entorno sociopolítico en que estas personas viven (Maxwell, 1998).

Por último, señalar, que los conflictos que surgen en la convivencia y socialización de los hijos en el seno de las parejas mixtas no responden tanto a las diferencias o incompatibilidades culturales como podría parecer a priori, sino más bien a factores 
socioeconómicos, situacionales y personales. Uno de los hechos que avalan esta tesis es que la mayoría de los problemas analizados en los matrimonios mixtos se reproducen con exactitud en los matrimonios endógamos (Rodríguez, 2004b: 184-185).

\section{Algunas aproximaciones metodológicas en el estudio de los matrimonios mixtos}

El estudio de Olson y Fowers (1993), aunque no abordan el análisis de los matrimonios mixtos, pretende lograr el desarrollo de una tipología empírica de matrimonios o parejas usando las puntuaciones del Positive Couple Agreement (PCA) procedentes de la escala multidimensional ENRICH. Esta aproximación metodológica, cuya unidad de análisis es la pareja, se basa en las diferencias relevantes entre parejas más que entre variables.

En cuanto a las características de la escala multidimensional ENRICH, las puntuaciones del PCA ENRICH proporciona una medida de la relación de pareja que se deriva de la combinación de las respuestas del compañero. Las puntuaciones de las parejas se denominan Positive Couple Agreement (PCA) porque mide el grado de acuerdo en la descripción de su relación en términos positivos con respecto a cada una de las escalas. La puntuación del PCA es el \% de ítems en una escala en que los acuerdos de la pareja caracterizarían aquellos aspectos de su relación en términos positivos (del 0 al 100\%).

La escala ENRICH contiene 125 ítems en 14 escalas. ENRICH está diseñada para identificar las fuerzas de las relaciones en 11 campos o esferas de relaciones: satisfacción matrimonial, cuestiones personales, comunicación, resolución de conflictos, gestión financiera, actividades de tiempo libre, relaciones sexuales, niños y crianza de los hijos, familia y amigos, roles equitativos y orientación religiosa. Cada una de estas escalas contiene 10 ítems. Además, ENRICH contiene tres escalas más que determinan la distorsión idealista, la cohesión de la pareja y la adaptabilidad ${ }^{6}$. Se introduce, a su vez, tres ítems como indicadores de la validez externa de la tipología: la medida de satisfacción matrimonial, la medida de divorcio potencial y el estado civil.

\footnotetext{
${ }^{6}$ Estas tres últimas escalas no se incluyen en el estudio en cuestión (Olson y Fowers, 1993).
} 
En cuanto a la metodología de análisis utilizada en el estudio (Olson y Fowers, 1993), los autores emplean tres etapas en el procedimiento del análisis cluster que finaliza con la obtención de cinco tipos de matrimonios o parejas que presentan diferencias significativas entre ellas: parejas desvitalizadas, parejas conflictivas, parejas tradicionales, parejas harmoniosas y parejas vitalistas.

Centrándonos en las investigaciones sobre el tema que nos concierne, el de los matrimonios mixtos, encontramos una diversidad de metodologías y técnicas utilizadas por los investigadores. En cuanto a los estudios realizados en los EEUU y, siguiendo con la tradición metodológica del país, la aproximación usada es de corte cuantitativo, en la cual la explotación de los datos del Censo de Población estadounidense constituye el procedimiento más habitual (Fun, 2001; Lee y Edmonston, 2005). Concretamente, Fun (2001) lleva a cabo un análisis exhaustivo de los datos del Censo de Población de 1990 a partir de los cuales el investigador estima modelos ${ }^{7}$ para matrimonios no mixtos, por un lado, y matrimonios mixtos de blancos con negros, con japoneses americanos y con mexicanos americanos, por otro. Por su parte, Lee and Edmonston (2005) se basan en el análisis de los datos procedentes de los censos desde 1970 hasta 2000 para examinar la evolución del fenómeno en los Estados Unidos y sus características.

Desde el punto de vista de la aproximación cuantitativa predominante en la literatura estadounidense, Kalmijn (1998: 404-406) nos ofrece una síntesis de las principales medidas y modelos que se han utilizado en los trabajos empíricos sobre matrimonios mixtos y endogamia. La medida más general utilizada para describir los matrimonios mixtos es el porcentaje de las parejas mixtas. Otras medidas utilizadas serían el "odds ratio", el índice de matrimonios mixtos y la correlación de Pearson. El segundo tipo de medidas utilizadas en los matrimonios mixtos son los modelos loglineales para describir los patrones de la selección matrimonial.

La investigación de Dan Rodríguez (2004) combina la metodología cualitativa con la metodología cuantitativa.

7 El autor utiliza los modelos log-lineales para analizar la clasificación cruzada de la educación de los maridos y las esposas con la etnia. 
La metodología cualitativa se basa principalmente en la observación participante y en entrevistas de dos tipos: a) entrevistas temáticas-prospectivas y b) entrevistas semi-dirigidas. Las primeras se aplicaron a individuos que disponen de mayor información, es decir, a representaciones de asociaciones de inmigrantes, así como a técnicos y representantes de las administraciones locales. Mientras que, las entrevistas de tipo semi-dirigida recogen información tanto del individuo o pareja objeto de estudio. Éstas se estructuraron en dos partes: la primera recogía información biográfica y socio-demográfica del individuo, de su cónyuge o pareja, padres, hermanos e hijos, particularmente en cuanto a las uniones y patrones de endogamia /exogamia: edad, lugar de nacimiento, grupo étnico, religión, ocupación, lugar de residencia, nivel educativo, relación de parentesco, número de hijos, etc. Esta información (de cuatro generaciones) se utilizó para el análisis genealógico. La segunda parte constituía la entrevista en profundidad propiamente dicha, centrada en las descripciones de las actitudes y comportamientos en torno a la endogamia y la exogamia.

Por otro lado, la investigación cuantitativa en Cataluña consistió en la aplicación de cuestionarios-bola de nieve "virtual" (muestreo por cuotas y cadenas de referencia) a

251 individuos. Esta cifra total incluía a los individuos/ parejas a los que se habían realizado entrevistas semidirigidas. El cuestionario contenía 51 variables, y recogía información sobre el individuo y sus uniones: lugar de nacimiento, edad, año de llegada, situación legal, religión, grupo étnico, estado civil, lugar de residencia, ocupación, salario, etc. Asimismo, el investigador lleva a cabo una explotación del Censo de Población de 1991 y de la Estadística de Población de Cataluña de 1996 correspondientes a la nupcialidad de cuatro poblaciones inmigradas en Cataluña: África (total), Gambia, Senegal y Marruecos.

Finalmente, la investigación de Santelli y Collet (2003) en Francia, propone una visión nueva de las parejas mixtas que toma en cuenta las dimensiones sociales y culturales. Se trata de una investigación realizada a partir de una población de referencia en la que al menos un cónyuge francés desciende de padres magrebíes. Dicha investigación constituye esencialmente un estudio cualitativo pero complementado con los resultados de la encuesta 'Etude de l'Histoire 
Familiale 1999". La parte cualitativa del estudio consistió en la aplicación de entrevistas tanto a las parejas como a los dos cónyuges de la pareja con el fin de estudiar los procesos de formación conyugal para cada uno de ellos. La investigación se articula alrededor de dos momentos de la vida conyugal: la formación de las parejas, a través de las modalidades de la elección conyugal y de la homogamia social y cultural, y las dinámicas conyugales, a través del nombre dado a los hijos $^{8}$ y de la cultura cotidiana "del otro".

\section{Metodología de la investigación}

La producción de la información a la que hace referencia esta investigación financiada por el Ministerio de Educación y Ciencia, se basa en el análisis de estudios de caso mediante entrevistas en profundidad semiestructuradas. Para ello se ha tomado como unidad de análisis las parejas o matrimonios mixtos (aquellos matrimonios en los que uno de los cónyuges tiene un origen cultural o su primera nacionalidad es diferente a la española).

La elección de la técnica de la entrevista en profundidad responde al hecho de permitir el establecimiento de cierto clima propicio para que emerjan aspectos relacionados con la descripción y valoración del grupo de pertenencia que de otra manera pudieran ser censurados en otro tipo de situaciones diseñadas por el investigador de tipo grupal (reuniones de grupo): la confidencialidad; la mayor facilidad de expresión de lo reservado dentro del juego público/ privado de la microsituación, ofrecen ventajas incuestionables para los objetivos de este estudio.

El muestreo aplicado es el intencional de "máxima variabilidad" establecido para alcanzar un grado importante de heterogeneidad controlada. Como señala Alaminos (1988: 90), este muestreo constituye una estrategia no probabilística válida para la recolección de datos, sobre todo en el caso de muestras pequeñas y específicas. En esta línea, Quinn (1988), citado en Alaminos (1988: 90) afirma que "la potencia del muestreo estadístico depende de seleccionar una muestra

${ }^{8}$ La elección del nombre del niño/a es significativo porque revela las posturas identitarias de la familia constituida (Santelli y Collet, 1998: 67). 
verdaderamente aleatoria y representativa que permita hacer generalizaciones desde la muestra a una población mayor. La potencia en el muestreo intencional está en seleccionar casos ricos en información para estudiar en profundidad”.

La variabilidad interna se ha definido en base a 4 niveles de control: nacionalidad, género, descendencia (con hijos/sin hijos) y un factor de asimetría, representado por el estatus construido en términos de ocupación y educación. Estas variables se emplean como factores explicativos para evaluar cómo los procesos de socialización, aculturación e identidad se desarrollan en el interior de una unidad familiar multicultural.

En cuanto al primer nivel de control, la nacionalidad, la finalidad es analizar la unidad familiar heterogénea controlada por la nacionalidad de origen: cómo se produce la transmisión de valores a la segunda generación.

En el segundo nivel de control de variabilidad se han considerado las diferencias de nacionalidad tanto en hombre como en mujeres. La finalidad es analizar las diferencias por género en función de los países de origen.

Por su parte, la variable hijos o no hijos permite evaluar de forma diferenciada la interacción con la segunda generación. Por un lado para analizar la posibilidad de conflictos en el interior de la pareja como consecuencia de los acuerdos o desacuerdos en la pautas de socialización, en la educación de los hijos, etc. A su vez, es posible que existan diferencias en las pautas de socialización, de construcción de identidades y en el proceso de aculturación según el género de los hijos.

Por último, el cuarto nivel de análisis (estatus-ocupación) estaría representado por un factor de asimetría para controlar la influencia del "poder" dentro de la unidad familiar. Este nivel se construye a partir de una variable combinada de educación y ocupación con el resultado de 4 categorías:

- Asimétrico padre: Mayor nivel del padre que de la madre (Ap);

- Asimétrico madre: Mayor nivel de la madre que del padre (Am); 
- Simétrico bajo padre y madre: Bajo nivel del padre y de la madre (Bmp)

- Simétrico alto padre y madre: Alto nivel del padre y de la madre (Amp)

El control de la asimetría permite una variabilidad de cuarto nivel para el control de poder dentro de la pareja, lo que posibilita controlar las diferencias de estatus en la pareja.

\section{Algunas reflexiones finales}

La consolidación de nuestro país como país de inmigración y la creciente diversificación étnica de los flujos migratorios establecidos en España trae consigo importantes implicaciones en diferentes niveles que suponen un desafío para nuestra sociedad. Una de las consecuencias es el incremento de la diversidad cultural en España y de los espacios multiculturales o interculturales. En este sentido, uno de los fenómenos que contribuyen al aumento en la creación de dichos espacios y procesos interculturales es la formación de las parejas y matrimonios mixtos. Los datos al respecto corroboran que se trata de una realidad creciente en nuestro país y que, por tanto, se requiere del análisis de este espacio complejo para comprender la sociedad hacia la cual nos encaminamos.

Tal y como señala Kalmijn (1998: 397), la relevancia sociológica del matrimonio mixto reside en su dinámica inherente: no solamente es una reflexión sobre las fronteras que actualmente separan los grupos en la sociedad, sino que también conduce a un potencial cambio socioeconómico y cultural. Es decir, la investigación sobre matrimonios mixtos nos revela cómo las sociedades modernas cambian.

De la revisión teórica realizada anteriormente destacamos en primer lugar, la importancia que adquieren algunas variables (edad, sexo, nivel educativo, clase social, lugar de residencia, tamaño del grupo, etc.) en la conformación de las parejas o matrimonios mixtos. En este sentido, cabe reiterar la preeminencia de los aspectos de clase social tanto en las uniones endógamas como las exógamas y la importancia del factor cultural en tales uniones. 
En segundo lugar, la consideración de la unión mixta como un indicio significativo de la integración debe ser cuestionada y relativizada, al depender muchas veces del contexto social, político y legislativo en que se enmarque, especialmente de este último factor.

Otro aspecto que cabe señalar, es el crecimiento de grupos de población con múltiples identidades a raíz del aumento de uniones mixtas y consecuentemente de familias multiculturales. En este sentido, la identificación cultural y/o étnica por parte de los hijos que viven en familias multiculturales será crucial para la futura configuración étnica y cultural del país.

De ahí que resulte imprescindible el análisis de la transmisión generacional de valores, creencias y modelos socio-culturales dentro la unidad familiar multicultural. Pues es en este contexto, de socializacion y educación de los hijos, donde tiene lugar el proceso de construcción de identidades en el entorno familiar. Se trata así, de un espacio complejo donde coexisten los procesos de cambio y mantenimiento cultural, conflicto y negociación, así como de transformación, redefinición y reelaboración de las prácticas y las identidades culturales. Estas dinámicas del mestizaje, por tanto, darían lugar a respuestas muy diversas que precisan ser observadas y analizadas. Enlazado con esta última idea, finalizamos resaltando la utilidad del estudio de los espacios interculturales y las dinámicas de mestizaje de las uniones mixtas para la construcción de espacios que permitan la convivencia, la integración y el respeto mutuo. 


\section{Bibliografía}

Alaminos, A. (1988). Teoría y práctica de la encuesta, Madrid, Cedeal.

Alba, R.D y Golden, R.M. (1986) "Patterns of ethnic marriage in the United States”. Social Forces, vol. 65, pp. 202-223.

Bastide, R. (1961) “Dusky Venus, Black Apollo", Race, vol. 3, n 1, pp. 10-18.

Becker, G. (1991) A Treatise on the Family. (Enlarged ed.), Cambridge, MA, Harvard University Press.

Bermúdez, E. (2007) Historias de unión y de amor en parejas mixtas que residen en la Comunidad V alenciana: relatos desde la masculinidad y la feminidad. Cuadernos de Investigación CEIM, n ${ }^{\circ} 3$.

Breger, R., y Hill, R., (eds.) (1998) Cross-cultural Marriage. Identity and Choice. Oxford, Berg.

Carabaña, J. (1983) "Homogamia y movilidad social", Revista de Investigaciones Sociológicas, $\mathrm{n}^{\mathrm{o}}$ 21, pp. 61-81.

Coleman, D. A. (1994) "Trends in fertility and intermarriage among immigrant populations in Western Europe as measures of integration". Journal of Biosocial Science, vol. 26, pp. 107-136.

Cortina, C; Esteve, A. y Domingo, A. (2006) "Crecimiento y singularidades demográficas de los matrimonios de extranjeros en España”. Migraciones, vol. 20, pp. 75-105.

Crester, G. (1990) "Intermarriage between 'White' Britons and Immigrants from the New Commonwealth and Pakistan". Journal of Comparative Family Studies, vol. 21, no 2, pp. 227-239.

Davis, K. (1941) "Intermarriage in Caste Societes". American Anthropologist, vol 43, pp. 376-395.

Delanty, G. (2003) Community. London, Routledge.

Edsmonston, B., Lee, S. y Passel, J. (2002) "Recent trends in intermarriage and immigration and their effects on the future racial composition of the US population" Perlmann, J. y Waters, M. (eds.) (2002) The new race question. How the census multiracial individuals. New York, Rusell Sage Foundation, pp. 227-255. 
Finnas, F. (1988) "The demographic Effect of Mixed marriages". European Journal of Population, vol. 4, n 2, pp. 145-156.

Fowers, B. J. y Olson, D. H. (1989) “The ENRICH inventory: A discriminant validity and cross-validation study", Journal of Marital and Family Therapy, no 15, pp. 65-79.

(1992), "Four types of premarital relationships: An empirical typology based on PREPARE", Journal of Family Psychology, nº 6, pp. 1021.

Fu, V. K. (2001) "Racial intermarriage pairings", Demography, vol 38, n 2 , pp.147-159.

Girard, A. (1981) [1964], Le Choix du conjoint. Une enquête psychosociologique en France. Travaux et documents, 70, París, INED-PUF.

Golstein, J. (1999) "Kindhip Ties thata Cross Racial Lines: The exception or the rule?", Demography vol. 36, n³, pp. 399-407.

Gonzalez-Ferrer, A. (2006) "Who Do Immigrants Marry? Partner Choice Among Single Immigrants in Germany". European Sociological Review, vol. 22, $\mathrm{n}^{\circ}$ 2, pp. 171-185.

INE (2007) Padrón Municipal de Habitantes, disponible en http://www.ine.es.

Kalmijn, M. (1991a) "From Family Origins to Individual Destinations", PhD dissertations, Departament of Sociology, UCLA.

(1991b) "Shifting selection among the children in religious and educational homogamy”. American Sociology Review, vol. 56, pp. 786-800.

(1998) "Intermarriage and Homogamy: Causes, Patterns and Trends". Annual Review of Sociology, vol. 24, pp. 395-421.

Kymlicka, W. (1996) Ciudadanía multicultural. Una teoría liberal de los derechos de las minorías. Barcelona, Paidos.

Lee, S. M. y Edmonston, B. (2005) "New Marriages, New Families: U.S. Racial and Hispanic Intermarriage". Population Bulletin, vol. 60, n², pp. 1-36.

Lievens, J. (1998) "Interethnic Marriage: Bringing in the context thought multilevel modelling". European Journal of Population, vol 14, pp. 117-155. 
(1999) "Family- Forming Migration from Turkey and Morocco to Belgium: The Demand for Marriage Partners from the Countries of Origin”. International Migration Review, vol. 33, n³, pp. 717-744.

Lustig, M. y Koester, J. (1999) Intercultural competence: interpersonal communication across cultures. Boston, Allyn and Bacon.

Lustig, M. y Koester, J. (eds.) (2000) Among Us. Essays on identity, belonging and intercultural competence. New York, Addison Wesley Longman.

Malgesini, G. y Giménez, C. (2000) Guía de conceptos sobre migraciones, racismo e interculturalidad. Madrid, Catarata.

Mare, M. (1991) "Five Decades of Educational Assortative Mating". American Sociological Review, vol. 56, pp. 15-32.

Maxwell, A. (1998) "Not all Sigues are Black or White: Some Voices from the Offspring of Cross-Cultural Marriages", Breger, R. y Hill, R. (eds.) (1998) Cross-cultural Marriage. Identity and Choice. Oxford, Berg.

Merton, R. (1941) "Intermarriage and the Social Structure: Fact and Theory", Psychiatry: A Journal of the Biology and the Pathology of Interpersonal Relations, vol. 4, pp. 361-374.

Ministerio de trabajo y asuntos sociales (Varios años), Anuario Estadístico de Extranjería. Madrid, Ministerio de Trabajo y Asuntos Sociales.

Murstein, BI (1976) Who Will Marry Whom: Theories and Research in Marital Choice. New York, Springer.

Musham, H. V. (1974) "The marriage squeeze", Demography, vol. 11, no2, pp. 291-299.

Neyrand, G. (1998) 'Y a-t-il des couples plus 'mixtes' que d'autres? De l'usage du terme mixité dans le domaine de la conjugalité". Dialogue, vol.139, nº1, pp.5-15.

Olson, D. H, Fournier, D. G. y Druckman, J. M. (1987), Counselor's manual for PREPARE/ ENRICH (rev. ed.), Minneapolis, MN: PREPARE/ ENRICH, Inc.

Olson, David H. y Fowers, Blaine (1993), "Five Types of Marriage: An Empirical Typology Based on ENRICH", The Family Journal, vol. 1, $\mathrm{n}^{\mathrm{o}}$ 3, pp. 196-215. 
Oppenheimer, V. (1997) “Women's Employment and the Gain to Marriage: The Specialization and Trading Model", Annual Review of Sociology, vol.23, pp.431-453.

Perlmann, J. (1997) "Multiracials, Intermarriage, Ethnicity". Society, vol. $34, n^{\circ} 6$, pp. $20-23$.

Perlmann, J. y Waters, M. (eds.) (2002). The new race question. How the census multiracial individuals. New Cork, Rusell Sage Foundation.

Perotti, A. (1989) Pequeño léxico sociológico y antropológico sobre la inmigración, Cuaderno n ${ }^{\circ}$ 65, Fundacion Encuentro, Servicio de Documentación.

Philippe, C., Varro, G., y Neyrand, G. (eds.) (1998) Liberté, égalité, mixité... conjugales. Une sociologie du couple mixte. Paris, Anthropos.

Rodrigo, M. (1997) "Elementos para una comunicación intercultural", Revista CIBOD d'Afers Internacionals, no 36.

Rodríguez, D. (2004a), "Inmigración y mestizaje hoy. Formación de matrimonios mixtos y familias transnacionales de inmigrantes en Cataluña”. Migraciones, vol.16, pp.77-120.

(2004b) Inmigración y mestizaje hoy. Formación de matrimonios mixtos y familias transnacionales de población africana en Cataluña, Barcelona, Publicaciones d'Antropologia Cultural, Universitat Autònoma de Barcelona.

Sandefur, G. (1986) “American Indian Intermarriage”. Social Science Research, vol.15, pp. 347-371.

Santelli, E. y Collet, B. (2003) "Comment repenser les mixités conjugales aujourd'hui? Modes de formation des couples et dynamiques conjugales d'une population française d'origine maghrébine". Revue Européenne des Migrations Internationales, vol. 19, n¹, pp. 51-79.

Streiff-Fenart, J. (1994) "Problèmes de terminologie et ambiguïté de la notion", Labat, C. y Vermes, G. (eds.) Cultures ouvertes sociétés interculturelles. Paris, L'Harmattan, pp. 226-232.

Taylor, CH. (1993) El multicuturalismo y la 'politica del reconocimiento'. Mexico DF, FCE.

Therborn, G. (1995). European Modernity and Beyond. The Trajectory of European Societies 1945-2000. Londres, Sage. 
Tognetti-Bordogna, M (1996). Legami familiari e immigraz̧ione: i matrimoni misti, Torino, L'Harmattan Italia.

Tucker, M. y Mitchell-Kernan, C. (1990) "New trends in Black American interracial marriage: The Social structural Context". Journal of Marriage and the Family, vol. 52, n'1, pp. 209-218.

Varro, G. (1994) 'Sur la construction de l'objet 'mariage mixte' en LABAT, C. y Vermes, G. (eds.) Cultures ouvertes sociétés interculturelles. Paris, L'Harmattan, pp. 213-219.

(1995) "La notion de 'mariage mixte", Varro, G. (ed.) Les couples mixtes et leurs enfants en France et en Allemagne. Paris, Armand Colin, pp. 29-51.

Walsh, C. (2001). La interculturalidad en la Educación. Lima, DINEBI.

Zonabend, F. (1981) "Le très proche et le pas trop loin. Reflections sur l'organization du champ matrimonial des societés à structures de parenté complexes ", Ethnologie Française, XI (4), pp. 311-3. 